\title{
Existence, uniqueness and regularity of the free boundary in the Hele-Shaw problem with a degenerate phase
}

\author{
Ivan A. Blank, Marianne K. Korten, and Charles N. Moore \\ Abstract. The Hele-Shaw model describes the flow of a viscous fluid being \\ injected into a slot between two nearby plates. It is used in injection molding \\ for the production of packaging materials and the interior plastic parts of cars \\ and airplanes, in electromechanical machining, and to study the diffusion of \\ nutrients and medicines within certain tumors. \\ We obtain the unique weak solution to the Hele-Shaw problem with a \\ mushy zone as the (pointwise) "Mesa" type limit of solutions to one-phase \\ Stefan problems with increasing diffusivities, and fixed initial and boundary \\ data. We discuss results on the regularity of the free boundary in space.
}

\section{Introduction}

The classical Hele-Shaw problem with Dirichlet data is usually formulated as: Given a bounded domain $D \in \mathbb{R}^{n}$ with smooth boundary $\partial D$, a finite set of closed curves $\left\{s_{0}^{j}(x)=0\right\}$ such that $D$ is contained in the union of their interiors, and a continuous function $p(x, t)$ defined on $\partial D \times(0, \infty)$, find $V(x, t)$ and a family of domains $S(t)$ (which each contain $D$ ) with $\partial S(t)=\{s(x, t)=0\}$ and such that

$$
\begin{array}{rlrl}
\Delta_{x} V & =0 & & (x, t) \in S(t) \backslash D \\
V(x, t) & =0 & & (x, t) \in \partial S(t) \\
V(x, t) & =p(x, t) & & (x, t) \in \partial D \times(0, \infty) \\
\nabla_{x} V \cdot \nabla_{x} s & =\frac{\partial s}{\partial t} & & (x, t) \in \partial S(t) \\
\partial S(0) & =\cup_{j}\left\{s_{0}^{j}(x)=0\right\} . &
\end{array}
$$

Sometimes the normal derivative of $V$ at the slot, $\partial D$, is prescribed, or curvature dependent terms are included in the free boundary condition.

Several questions concerning the Hele-Shaw problem have been open for some time. Among them: to find a workable weak formulation, to find tools to address the regularity of solutions and interfaces in time (since in the classical formulation time dependence is only "visible" in the free boundary condition), and whether

1991 Mathematics Subject Classification. Primary 76D27; Secondary 35K65, 49J40.

Key words and phrases. Mesa problem; Hele-Shaw problem; Stefan problem; free boundary; mushy region.

I. Blank is partially supported by NSF grant \#0501770. M. Korten is partially supported by NSF EPSCoR grant \#530517 under agreement NSF32169/KAN32170 and NSF grant \#0503914. 
uniquenss would hold. There has long been a feeling that there should be some relation between the Hele-Shaw and the Stefan problems, and that "mushy" regions should be expected in the Hele-Shaw problem.

In this contribution we discuss a weak formulation of the Hele-Shaw problem which allows for a mushy region, and provides the classical solution if the free boundary and the solution are regular enough. We show that the solution to this problem can be approximated as a mesa - type limit by solutions of one-phase Stefan problems with increasing diffusivities, and with the initial and boundary data held fixed. Much is known about the behavior of solutions of the approximating problems, and we exploit this to derive information about the behavior of the solution to the Hele-Shaw problem. Additional benefits are that our method does not require the injection slot to be connected (interesting for applications where multiple slots may be needed), and that the limiting weak fomulation as a parabolic equation enjoys uniqueness under quite general conditions.

In $[\mathbf{B K M}]$ we first assume the Dirichlet data at the injection slot are nondecreasing in $t$. Since this assumption appears and disappears frequently throughout this note, for brevity we will designate this assumption as (PNDT): $p(x, t)$ is nondecreasing in $t$, for $x \in \partial D \times(0, \infty)$. [BKM] also deals with the case when (PNDT) is not assumed, and this paper will compare and contrast these situations.

The mesa problem describes the limit of solutions $u_{m}$ of, say, the porous medium equation, with fixed initial data. It first appeared in connection with the modeling of problems related to transistors (see $[\mathbf{E H K O}]$ ). Caffarelli and Friedman $[\mathbf{C F}]$ studied the initial value problem in $\mathbb{R}^{n} \times[0, \infty)$, showing the limit exists, is independent of the chosen subsequence, is independent of time, that it is equal to the characteristic function of a set plus the initial data times the characteristic function of the complement of that set, and finally that this set can be characterized as the noncoincidence set of a variational inequality. Further developments showed the same conclusions hold when $u_{m}$ is replaced by a fairly general sequence of monotone constitutive functions $\phi_{m}(u)$ with $\phi_{m}(0)=0$ (see $[\mathbf{F H}]$ ), moreover, this behavior is a property of fairly general semigroups (see $[\mathbf{I}]$, [BEG]). A mesa problem for an equation giving rise to a mushy region was studied in [BoKM]. Here we show all of the properties shown by $[\mathbf{C F}]$ mentioned, except our limits will not be independent of time. (This evolution in time is natural since we work in an outer domain where the inner boundary data is a source.)

Our results should be compared with the nice results in [GQ1] and [GQ2]. We will use the same notion of weak solution to the Hele-Shaw problem, but instead approximate the solution $(u, v)$ with $\left(u_{m}, m(u-1)_{+}\right)$where $u_{m}$ is a sequence of solutions to the one-phase Stefan problem with increasing diffusivities. (See also $[$ GQV] for other approximation results.) Rather than using a sequence of pressures and the one-sided inequality we use natural energy estimates for the Stefan problem to obtain compactness. Our different approach gives some advantages: it seems to shorten the treatment, and allows for better convergence in the case of constant boundary data, as well as still yielding compactness for any nonnegative boundary data. We use regularity for the obstacle problem to deduce spatial regularity, although it is quite likely this part of our argument could be used to deduce spatial regularity from their results. It has been long suspected that the Stefan and HeleShaw problem should be naturally related; in this paper we shed light on this relationship. 
We exploit a mesa limit setting in an outer domain $D^{c}$ to obtain naturally a weak formulation of the Hele-Shaw problem with Dirichlet data as in (1.1). The use of one-phase Stefan problems with mushy regions and increasing diffusivities naturally produces a mushy region when the initial data $u_{I}$ take values in $[0,1]$. An advantage of this approach is that changes in the topology of the "wet" region do not interfere with the construction. In short, whereas other authors (e.g. see [DL]) make a priori assumptions to ensure the free boundary stays smooth, we show existence of weak solutions for all time, regardless of possible changes in topology. Indeed, there are very natural problems arising in applications where the topology changes. Consider, for example, flow around an obstacle: there is a change in topology when the flow meets itself on the other side.

1.1. Definition (m-approximating problem-classical version). Let $u^{(m)}$ solve:

$$
u_{t}^{(m)}=m \Delta\left(u^{(m)}(x, t)-1\right)_{+}, \quad(x, t) \in D^{c} \times(0,+\infty) \cap\left\{u^{(m)}>1\right\},
$$

with initial and boundary data given by

$$
\left.\begin{array}{rlrl}
u^{(m)}(x, 0) & =u_{I}(x), & & x \in D^{c}, \\
m\left(u^{(m)}(x, t)-1\right)_{+} & =p(x, t), & & (x, t) \in \partial D \times(0,+\infty),
\end{array}\right\}
$$

and with the free boundary condition

$$
\left(-\nabla_{x} m\left(u^{(m)}-1\right)_{+},\left(1-u_{I}\right)\right) \cdot \nu=0, \quad(x, t) \in \partial\left\{u^{(m)}>1\right\} .
$$

Here $\nu$ is the outer $(\mathrm{n}+1)$ dimensional normal to $\left\{u^{(m)}>1\right\}$; (1.4) will be satisfied when the free boundary is smooth. The weak formulation we will give will not require any regularity assumptions on the free boundary or initial data $u_{I}$. We assume $0 \leq u_{I} \leq 1$ has compact support, $0<p(x, t) \in C^{2, \alpha}$ and $D$ is bounded with $\partial D \in C^{2, \alpha}$. Our assumption $u_{I}$ has compact support is only technical and simplifies the construction of certain comparison functions, but it is not necessary. As to assuming $p(x, t)>0$, note $p(x, t) \equiv 0$ leads to no evolution. To see this, extend $u^{(m)}$ by 1 across $D$. Thus, positivity of $p(x, t)$ drives the evolution.

In this formulation $u^{(m)}$ is energy (or enthalpy), $m\left(u^{(m)}-1\right)+$ is temperature and (1.2) expresses conservation of energy. As $m$ increases, the diffusion accelerates; competing is the decrease to 1 of the boundary data for $u^{(m)}$ on $\partial D$.

I. Blank thanks Kansas State University for their hospitality during much of this work. M. Korten is indebted to Juan Luis Vázquez for introducing her to the Hele-Shaw problem. Korten and Blank acknowledge Kansas EPSCoR which supported Blank's visits.

\section{A weak formulation}

For our m-approximating problem, we consider an appropriate weak formulation:

2.1. Definition (Weak solutions of the m-approximating problem). A nonnegative $u^{(m)} \in L_{l o c}^{1}$ is a weak solution of the m-approximating problem if for any $\varphi \in C^{\infty}\left(\mathbb{R}^{n} \times[0, \infty)\right)$ such that $\varphi \equiv 0$ on $\partial D \times[0, \infty)$, and $\varphi(x, t) \rightarrow 0$ as either $t \rightarrow \infty$ or $|x| \rightarrow \infty$ we have

$$
\begin{aligned}
& \int_{D^{c}} \int_{0}^{\infty} \varphi_{t}(x, t) u^{(m)}(x, t) d t d x+\int_{D^{c}} \int_{0}^{\infty} \Delta_{x} \varphi(x, t) m\left[u^{(m)}(x, t)-1\right]_{+} d t d x \\
& =\int_{\partial D} \int_{0}^{\infty} \frac{\partial \varphi}{\partial \nu}(x, t) p(x, t) d t d \mathcal{H}^{n-1}(x)-\int_{D^{c}} \varphi(x, 0) u_{I}(x) d x
\end{aligned}
$$


We observe that the traces of $u^{(m)}$ and $m\left(u^{(m)}-1\right)_{+}$on the boundaries of our domain will be well-defined. (See $[\mathbf{K} \mathbf{1}]$ which adapts results from $[\mathbf{D K}]$, and Lemma 3.2 of [K2].) Observe that the classical formulation (i.e. Equations (1.2) - (1.4) ) will be satisfied whenever the functions and sets are sufficiently smooth. (See [Bo2] for (1.4) .) We cannot expect $u^{(m)}$ to be continuous across the free boundary, however $m\left(u^{(m)}-1\right)_{+}$is continuous. (See $[\mathbf{K} \mathbf{1}]$ which verifies the required assumptions of [DB] for continuity.) Concerning the behavior of $u^{(m)}$ in $t$, we have:

2.2. TheOREM. Almost every $x$ has the property: if $0 \leq u^{(m)}(x, t) \leq 1$ for a.e. $t \in(a, b)$, then $u^{(m)}(x, t)$ is constant on $(a, b)$.

Proof. Let $\eta(t)$ be $C^{\infty}$ and supported in $(a, b)$. Put $k(x)=\int_{a}^{b} \eta(t) m\left(u^{(m)}-\right.$ $1)_{+}(x, t) d t, h(x)=\int_{a}^{b} \eta^{\prime}(t) u^{(m)}(x, t) d t$. Then $k$ is continuous and $\Delta k=h$ weakly and thus, since $\Delta k=0$ a.e. on any set where $k$ is constant, this holds at a.e. $x$ where $0 \leq u^{(m)}(x, t) \leq 1$. At such $x$, then $h(x)=0$. By considering an appropriate countable family of such $\eta$, we conclude $u^{m}(x, t)$ is constant in $(a, b)$. See [BKM] for complete details.

2.3. Remark. Lemma 4.2 of $[\mathbf{K 1}]$ shows that if $u^{(m)}(x, t)<1$ on a set $E$, then $u^{(m)}\left(x, t_{1}\right) \leq u^{(m)}(x, t)$ a.e. on $E$ whenever $t_{1}<t$. Combining the theorem with this fact and the continuity of $m\left(u^{(m)}-1\right)_{+}$tells us that at a.e. $x$, the behavior of $u^{(m)}(x, t)$ as $t$ increases is as follows: it starts at $u_{I}(x)$ and remains constant until it (possibly) jumps to 1; it then (possibly) exceeds 1 , and remains in the diffusive region forever after. In fact, the maximum principle shows it must remain strictly greater than 1.

Set $M:=\|p\|_{L^{\infty}}$. Use the maximum principle the equation enjoys to assert

$$
0 \leq u^{(m)} \leq 1+\frac{M}{m} \quad \text { or } \quad 0 \leq m\left(u^{(m)}-1\right)_{+} \leq M,
$$

and hence there exists a weak-* $L^{\infty}$ limit $u^{(\infty)}$ of the $u^{(m)}$ along a subsequence of $m$. It is immediate that $0 \leq u^{(\infty)} \leq 1$. We can take a further subsequence to ensure that $m\left(u^{(m)}-1\right)_{+}$has a weak- $* L^{\infty}$ limit $V$.

2.4. Remark. Equation (2.2) and remark (2.3) show that the essential range of $u^{(m)}(x, \cdot)$ is a subset of $\left\{u_{I}(x)\right\} \cup[1,1+M / m]$.

Taking limits in (2.1) gives:

2.5. Theorem. We have $0 \leq V \leq M$, and the pair $\left(u^{(\infty)}, V\right)$ satisfies

$$
\begin{aligned}
& \int_{D^{c}} \int_{0}^{\infty} \varphi_{t}(x, t) u^{(\infty)}(x, t) d t d x+\int_{D^{c}} \int_{0}^{\infty} \Delta_{x} \varphi(x, t) V(x, t) d t d x \\
= & \int_{\partial D} \int_{0}^{\infty} \frac{\partial \varphi}{\partial \nu}(x, t) p(x, t) d t d \mathcal{H}^{n-1}(x)-\int_{D^{c}} \varphi(x, 0) u_{I}(x) d x
\end{aligned}
$$

for any $\varphi \in C^{\infty}\left(\mathbb{R}^{n} \times[0, \infty)\right)$, such that $\varphi \equiv 0$ on $\partial D \times[0, \infty)$, and $\varphi(x, t) \rightarrow 0$ as either $t \rightarrow \infty$ or $|x| \rightarrow \infty$.

2.6. Definition. A pair $\left(u^{(\infty)}, V\right)$ satisfying (2.3) will be called a weak solution of the Hele-Shaw problem with boundary data $p(x, t)$ and initial data $u_{I}(x)$. 
2.7. Remark. The classical formulation (1.1) does not allow for a "mushy" zone- a region with positive energy, insufficient to initiate a change of phase. The weak formulation (2.3) easily accomodates for the existence of a mushy zone, which is one of the gains of formulating the problem as a limit of parabolic problems.

If we choose $\varphi$ to be compactly supported in $D^{c} \times(0, T)$ we see that the pair $\left(u^{\infty}, V\right)$ is a distributional solution of $\Delta_{x} V=u_{t}^{(\infty)}$. As we will see, $u^{(\infty)}=1$ in the region occupied by the fluid so that, in the weak sense, $\Delta_{x} V=0$ there.

2.8. TheOREM (Uniqueness). The solution of the limiting problem of Lemma (2.5) is unique, so all solutions of the Hele-Shaw problem as given in Definition (2.6) are recoverable via this Mesa limit process.

Proof. This follows from the uniqueness theorem in section 3 of [Bo].

2.9. Remark. Note our definition generalizes that found in $[\mathbf{D F}]$. Also, given our assumptions, $V(x, t)$ is not necessarily continuous in time. For example, consider the radially symmetric situation where $D=B_{1}$ and $u_{I}(r)=1$ on $3 \leq r \leq 5$, but is smaller outside. When what we want to call the free boundary reaches $r=3$, it will instantaneously jump to $r=5$, and this leads to an immediate jump in $V$.

2.10. Definition (The free boundary). We set

$$
t_{\infty}(x):=\inf \{t: V(x, t)>0\} \text { and } \tau_{\infty}(x):=\inf \left\{t: u^{(\infty)}(x, t)=1\right\} .
$$

We define the diffusive region at time $t$ for the m-approximating problem and the limiting Hele-Shaw problem to be respectively,

$$
\begin{aligned}
A^{(m)}(t) & :=\left\{x \in D^{c}: m\left(u^{(m)}(x, t)-1\right)_{+}>0\right\} \\
A(t) & :=\left\{x \in D^{c}: V(x, t)>0\right\} .
\end{aligned}
$$

Now we simply define the free boundary at time $t$ to be $F B^{(m)}(t):=\partial A^{(m)}(t) \backslash \partial D$, or $F B(t):=\partial A(t) \backslash \partial D$, according to which problem we are considering.

Because of the example in Remark (2.9) we conclude that the free boundary cannot be expected to vary continuously in time. It is clear that $\tau_{\infty}(x) \leq t_{\infty}(x)$, but they need not be equal. In the example, they differ on $3 \leq r \leq 5$.

\section{Monotonicity, inclusions, and consequences}

3.1. Lemma (Monotonicity of $\left.u^{(m)}\right)$. Assuming (PNDT), we have that for each $m>0$ and any $x \in D^{c}, u^{(m)}(x, t)$ is an increasing function of time.

Proof. If $u^{(m)}(x, t)<1$, this follows from Lemma 4.2 of [K1]. We conclude that as $t$ increases the $A^{(m)}(t)$ are nested nondecreasing sets. The idea of the rest of the proof is to consider $S:=\left\{(x, t): u^{(m)}(x, t)>1\right\}$, and then apply the weak maximum principle to the caloric function

$$
U(x, t):=u^{(m)}(x, t+\epsilon)-u^{(m)}(x, t)
$$

on $S$. (Observe that $U \geq 0$ on $\partial S$, in particular on $\partial D \times(0, \infty)$.)

The monotonicity of $u^{(m)}$ and $u^{(m)} \rightarrow u^{(\infty)}$ weak-* $L^{\infty}$ implies:

3.2. Lemma (Monotonicity of $\left.u^{(\infty)}\right)$. For a.e. $x, u^{(\infty)}(x)$ is monotone increasing in $t$. 
Now without data on the slot which compete with the increasing diffusivity (or if the functions solve the equation on all of $\mathbb{R}^{n}$ ), it is immediate by rescaling that the diffusive regions, $A^{(m)}(t)$, must be nondecreasing with $m$. In our case, however, we need to produce an appropriate barrier.

3.3. Theorem (Temperature increases with $m$.). Assuming (PNDT), if $m<k$, then

$$
m\left[u^{(m)}-1\right]_{+} \leq k\left[u^{(k)}-1\right]_{+} .
$$

Consequently, the $A^{(m)}(t)$ are nested: $m<k$ implies $A^{(m)}(t) \subset A^{(k)}(t)$.

Proof. We construct a subsolution of the k-approximating problem:

$$
v^{(k)}(x, t):= \begin{cases}u^{(m)}(x, t) & \text { if } u^{(m)}(x, t)<1 \\ 1+\frac{m}{k}\left(u^{(m)}(x, t)-1\right) & \text { if } u^{(m)}(x, t) \geq 1\end{cases}
$$

That this is a subsolution follows quickly from two easily verifiable statements: 1 . $\left\{v^{(k)}>1\right\}$ is identical to $\left\{u^{(m)}>1\right\}$ and $2 . k\left(v^{(k)}-1\right)_{+} \equiv m\left(u^{(m)}-1\right)_{+}$. (Only the definition of $v^{(k)}$ is needed to verify these!) By 1 . the free boundaries of $v^{(k)}$ and $u^{(m)}$ are identical in time and space, so the speed at every point is also identical. However, 2. quickly leads to the conclusion that $v^{(k)}$ satisfies the free boundary condition and the boundary value condition on the slot for the k-approximating problem exactly. Finally, we compute:

$$
v_{t}^{(k)}(x, t) \leq u_{t}^{(m)}(x, t)=m \Delta\left(u^{(m)}(x, t)-1\right)_{+}=k \Delta\left(v^{(k)}(x, t)-1\right)_{+}
$$

showing $v^{(k)}$ is a local subsolution to the $\mathrm{k}$-approximating problem, and therefore $v^{(k)}(x, t) \leq u^{(k)}(x, t)$. (The first inequality above uses Lemma (3.1) .) Thus,

$$
k\left[u^{(k)}-1\right]_{+} \geq k\left[v^{(k)}-1\right]_{+} \equiv m\left[u^{(m)}-1\right]_{+} .
$$

Consequently, the sequence $\left\{m\left[u^{(m)}-1\right]_{+}\right\}$is increasing and bounded. Hence:

3.4. Corollary (Pointwise convergence of temperature). Assuming (PNDT), then $\left\{m\left[u^{(m)}-1\right]_{+}\right\}$converges pointwise a.e. to $V$. In particular, $V$ is unique. (No subsequence is needed.)

3.5. CoROllary (Representation of $\left.u^{(\infty)}\right)$. There is an increasing (in the sense of set inclusion) set-valued function of $t$ which we call $Q(t)$ such that $u^{(\infty)}(x, t)$ admits the representation for almost every $(x, t)$ :

$$
u^{(\infty)}(x, t)=\chi_{Q(t)}(x)+u_{I}(x) \chi_{Q(t)^{c}}(x) .
$$

Furthermore, $Q(t)$ can be chosen to be equal to $\left\{x \in \mathbb{R}^{n}: \tau_{\infty}(x)<t\right\}$. If (PNDT) is assumed, then $u^{(\infty)}(x, t)$ is the pointwise limit a.e. of the $u^{(m)}(x, t)$. If (PNDT) is not assumed, then $u^{(m)}(x, t)$ converges in measure to $u^{(\infty)}(x, t)$.

Proof. We prove only the case where we assume (PNDT); otherwise see $[\mathbf{B K M}]$. From Lemma $(3.2), u^{(\infty)}(x, t)$ is an increasing function of $t$, so by Remark (2.4) $u_{I}(x) \leq u^{(\infty)}(x, t) \leq 1$. Thus, to prove (3.6) it suffices to show $u^{(\infty)}(x, \cdot)$ does not attain values strictly between $u_{I}(x)$ and 1 for a.e. $x$. Indeed, by combining the previous theorem with Remark (2.3) we see that the limit of $u^{(m)}(x, t)$ as $m \rightarrow \infty$ exists for a.e. $(x, t) \in \mathbb{R}^{n+1}$ and is either 1 or $u_{I}\left(x_{0}\right)$. It is a simple exercise to show the pointwise and weak- $* L^{\infty}$ limits coincide a.e. 
In terms of the "choice" of $Q(t)$, it is clear that the only $x$ where there can be a choice is on the (possibly empty) set $\left\{x \in \mathbb{R}^{n}: u_{I}(x)=1\right\}$. Examination of the definition of $Q(t)$ combined with the monotonicity of $u^{(\infty)}(x, t)$ in $t$ makes it clear that $Q(t):=\left\{x \in \mathbb{R}^{n}: \tau_{\infty}(x)<t\right\}$ will suffice. (Note that an equally good choice is $Q(t):=\left\{x \in \mathbb{R}^{n}: t_{\infty}(x)<t\right\}$.) With either choice $A(t) \subset Q(t)$.

3.6. REMARK. The set $Q(t)$ is simply the region into which the fluid has spread. In the region where the fluid has not yet spread, $Q(t)^{c}$, there is fluid standing with height $u_{I}(x)$.

3.7. REMARK. We note in either case (i.e. with or without (PNDT)), our convergence is strong enough to be able to invoke dominated convergence. We also note that the uniqueness of the solution to the limiting equation allows us to conclude that taking subsequences is unnecessary for any of the convergences we give.

3.8. Corollary ( $V$ is harmonic in the diffusive region). $\Delta_{x} V=0$ in int $A(t)$.

Although $V$ is discontinuous in general, we do get regularity in space.

3.9. Theorem (Spatial continuity of $V$ ). Assuming $(P N D T), V(\cdot, t)$ is lower semicontinuous. Also, for a.e. $t, V(\cdot, t)$ has a representative which is upper semicontinuous.

Proof. The $m\left[u^{(m)}(\cdot, t)-1\right]_{+}$are continuous and increase to $V(\cdot, t)$. Hence $V(\cdot, t)$ is lower semicontinuous. By Lemma $(3.2), d \nu=u_{t}^{(\infty)}$ is a nonnegative measure which can be "sliced" into Radon measures of one dimension less for a.e. $t$. (See $[\mathbf{M}]$ p. 139 - 142, and Equation (10.3) on p. 140 in particular.) Call these slices $d \nu_{t}$. Then for a.e. $t, \Delta_{x} V(x, t)=d \nu_{t}$, so $V(\cdot, t)$ is subharmonic. For such a $t, V(x, t)$ is upper semicontinuous at every $x$ which is a Lebesgue point of $V(x, t)$.

3.10. Remark. We would like to rule out two trivial cases: The possibility that the free boundary moves to infinity as soon as $t>0$, and the possibility it never moves. This can be done by producing a family of super- and subsolutions to our approximating problems whose free boundaries move in a suitable way, independent of $m$, and then using comparison principles. See [BKM] for details.

\section{Spatial regularity results}

Apply the Baiocchi transformation to $V(x, t)$ :

$$
W(x, t):=\int_{0}^{t} V(x, s) d s .
$$

Observe that by Lemma (3.2) (the diffusive regions increase in time) and by the positivity of $V(x, t)$ in the diffusive region, it follows that $\{W>0\}$ is identical to $\{V>0\}$. To find the regularity of $\partial\left\{x \in \mathbb{R}^{n}: W(x, T)>0\right\}$ we show $W(\cdot, t)$ belongs to $H_{l o c}^{1}\left(D^{c}\right)$ for a.e. $t$, and then that $W(x, t)$ is a weak solution of the following obstacle problem in a.e. time slice $t=T$

$$
0 \leq W(x, T), \quad \Delta_{x} W(x, T)=\chi_{\{W(x, T)>0\}}(x)\left(1-u_{I}(x)\right) .
$$

We may then invoke regularity results for the obstacle problem. (See $[\mathbf{B l}]$ and $[\mathbf{C}]$ and references therein.) For more detail on the results in this section see $[\mathbf{B K M}]$.

For simplicity, set $\alpha_{m}(s):=m(s-1)_{+}$. 
4.1. Lemma (Energy estimates for the $\left.u^{(m)}\right)$. Let $0<r<R$ and $0 \leq t_{0}<t_{1}$, and assume (PNDT) holds. Then there is a constant $C=C(n)(R-r)^{-2}$ such that:

$$
\int_{t_{0}}^{t_{1}} \int_{B_{r}\left(x_{0}\right)}\left|\nabla \alpha_{m}\left(u^{(m)}\right)\right|^{2} d x d t \leq C \int_{t_{0}}^{t_{1}} \int_{B_{R}\left(x_{0}\right)} \alpha_{m}\left(u^{(m)}\right)^{2} d x d t .
$$

4.2. REMARK. Note that the integral on each side has the same time interval. This nice property seems to be particular to the case where (PNDT) is assumed. For the energy estimates in the general case, see $[\mathbf{B K M}]$.

The proof just involves integrations by parts and is delicate but standard. See $[\mathbf{B K M}]$. Because $C$ is independent of $t$, we have:

4.3. Theorem (Time-slice energy estimates). If (PNDT) holds, then for a.e.t,

$$
\int_{B_{r}\left(x_{0}\right)}|\nabla V(x, t)|^{2} d x \leq C \int_{B_{R}\left(x_{0}\right)} V(x, t)^{2} d x,
$$

where once again, the constant $C$ has the form given in (4.1) .

Proof. Let $t_{1} \rightarrow t_{0}$ in the previous lemma and use the $L^{2}$ convergence of $\alpha_{m}\left(u^{(m)}\right)$ to $V$ and the lower semicontinuity of the Dirichlet integral.

4.4. Corollary. For a.e. $t, V(\cdot, t) \in H_{l o c}^{1}\left(D^{c}\right)$.

Let $K \subset D^{c}$ be compact. Since $W(\cdot, t) \leq M t$, we have $W(\cdot, t) \in L^{\infty}(K) \subset$ $L^{2}(K)$. Consider $\varphi \in C_{0}^{1}(K)$. By expressing $W$ as in (4.1) in terms of $V$, and $V$ as the limit of the $\alpha_{m}\left(u^{(m)}\right)$, carefully interchanging limits and using the energy estimates for the $\alpha_{m}\left(u^{(m)}\right)$, it can be shown that $\left(\frac{\partial W}{\partial x_{i}}, \varphi\right) \leq C t^{1 / 2}\|\varphi\|_{L^{2}(K)}$. Hence also $\frac{\partial W}{\partial x_{i}}(\cdot, t) \in L^{2}(K)$ and we have:

4.5. Theorem. For all $t>0, W(\cdot, t) \in H_{l o c}^{1}\left(D^{c}\right)$.

4.6. REMARK. In fact, once we show $W(\cdot, t)$ is a solution of the obstacle problem, elliptic regularity theory implies $W(\cdot, t)$ is $C^{1, \alpha}$ in space for all $\alpha<1$. Indeed, as a solution to the obstacle problem, $W$ will enjoy $C^{1,1}$ regularity (which is optimal here) assuming $u_{I}$ is Dini continuous. Even if $u_{I}$ is not Dini continuous, $W$ will still be bounded by a constant times the square of the spatial distance to the free boundary.

To derive Equation (4.2) we need to commute the Laplacian with the integral in time. To accomplish this, we turn back to the approximating problem. Using weak-* $L^{\infty}$ convergence of the temperature functions we have

$$
\lim _{m \rightarrow \infty} \int_{D^{c}} \int_{0}^{T}\left[\Delta_{x} \psi(x)\right] m\left[u^{(m)}(x, t)-1\right]_{+} d t d x=\int_{D^{c}}\left[\Delta_{x} \psi(x)\right] W(x, T) d x
$$

whenever $\psi$ is $C^{\infty}$ of compact support in $D^{c}$. On the other hand, by dominated convergence, for a.e. $T$

$$
\begin{aligned}
\lim _{m \rightarrow \infty} \int_{D^{c}} \psi(x)\left[u^{(m)}(x, T)-u_{I}(x)\right] d x & =\int_{D^{c}} \psi(x)\left[u^{(\infty)}(x, T)-u_{I}(x)\right] d x \\
& =\int_{D^{c}} \psi(x) \chi_{\{W(x, T)>0\}}(x)\left(1-u_{I}(x)\right) d x
\end{aligned}
$$

Since $W \geq 0$, we can combine the last two computations with the previous lemma to conclude that $W(\cdot, t)$ solves the obstacle problem for a.e. $t>0$. Equation 
(4.2) and this fact allows us to use $[\mathbf{B l}]$ and $[\mathbf{C}]$ to infer optimal regularity of the free boundary as long as we satisfy the nondegeneracy condition $u_{I}(x) \leq \lambda<1$.

\section{References}

[AK] D. Andreucci and M. Korten, Initial traces of solutions to a one-phase Stefan problem in an infinite strip, Rev. Mat. Iberoamericana, 9(1993), no. 2, 315-332.

$[\mathrm{BBH}] \mathrm{Ph}$. Bénilan, L. Boccardo, and M.A. Herrero, On the limit of solutions of $u_{t}=\Delta u^{m}$ as $m \rightarrow \infty$, Rend. Mat. Univ. Pol. Torino, Fascicolo Speciale (1989), Nonl. PDE's, 1-13.

[BEG] Ph. Bénilan, L.C. Evans, R.F. Gariepy, On some singular limits of homogeneous semigroups, J. Evol. Eq., 3(2003), no. 2, 203-214.

[Bl] I. Blank, Sharp results for the regularity and stability of the free boundary in the obstacle problem, Indiana Univ. Math. J. 50(2001), no. 3, 1077-1112.

[BKM] I. Blank, M.K. Korten, and C.N. Moore, The Hele-Shaw problem as a mesa limit of Stefan problems: existence, uniqueness and regularity of the free boundary, submitted, preprint available at http://arxiv.org/PS_cache/math/pdf/0410/0410131.pdf

[Bo] J.E. Bouillet, Nonuniqueness in $L^{\infty}$ : An example, Lecture notes in pure and applied mathematics, Vol. 148, Marcel Dekker, Inc., (1993), 35-40.

[Bo2] J.E. Bouillet, Signed solutions to diffusion heat conduction equations, in Free Boundary Problems: Theory and Applications, Proc. Int. Colloq., Irsee, Germany 1987, Vol II, Pitman Res. Notes Math. Series 186(1990), 888-892.

[BoKM] J.E. Bouillet, M.K. Korten, and V. Márquez, Singular limits and the "Mesa" problem, Rev. Un. Mat. Argentina, 41(1998), no. 1, 27-40.

[C] L.A. Caffarelli, The obstacle problem revisited, J. Fourier Anal. Appl., 4(1998), no. 4-5, 383-402.

[CF] L.A. Caffarelli and A. Friedman, Asymptotic behavior of solutions of $u_{t}=\Delta u^{m}$ as $m \rightarrow \infty$, Indiana Univ. Math. J., 36(1987), no. 4, 203-224.

[DK] B.E.J. Dahlberg and C.E. Kenig, Weak solutions of the porous medium equation, Trans. Am. Math. Soc., 336(1993), no. 2, 711-725.

[DL] P. Daskalopoulos and K. Lee, All time smooth solutions of the one-phase Stefan problem and the Hele-Shaw flow, Comm. PDE, 29(2004), no. 1-2, 71-88.

[DB] E. DiBenedetto, Continuity of weak solutions to certain singular parabolic equations, Ann. Mat. Pura Appl., (4), CXXX(1982), 131-176.

[DF] E. DiBenedetto and A. Friedman, The ill-posed Hele-Shaw model and the Stefan problem for supercooled water, Trans. Amer. Math. Soc., 282(1984), no. 1, 183-204.

[EHKO] C.M. Elliott, M.A. Herrero, J.R. King, and J.R. Ockendon, The mesa problem: Diffusion patterns for $u_{t}=\nabla \cdot\left(u^{m} \nabla u\right)$ as $m \rightarrow+\infty$, IMA J. Appl. Math., 37(1986), no. 2, 147-154.

$[\mathrm{FH}]$ A. Friedman and S. Huang, Asymptotic behavior of solutions of $u_{t}=\Delta \phi_{m}(u)$ as $m \rightarrow \infty$ with inconsistent initial values, Analyse mathématique et applications, Gauthier-Villars, Paris, (1988), 165-180.

[GQ1] O. Gil and F. Quirós, Convergence of the porous media equation to Hele-Shaw, Nonlinear Anal., 44(2001), 1111-1131.

[GQ2] O. Gil and F. Quirós, Boundary layer formation in the transition from the porous media equation to a Hele-Shaw flow, Ann. Inst. H. Poincaré Anal. Non Linéaire 20(2003), no.1, $13-36$.

[GQV] O.Gil, F. Quirós, and J.L. Vázquez, Zero specific heat limit and large time asymptotics for the one-phase Stefan problem, preprint.

[I] N. Igbida, The mesa-limit of the porous-medium equation and the Hele-Shaw problem, Diff. Int. Eqns., 15(2002), no. 2, 129-146.

[KN] D. Kinderlehrer and L. Nirenberg, Regularity in free boundary problems, Ann. Scuola Norm. Sup. Pisa Cl. Sci., (4)4(1977), no. 2, 373-391.

[KLV] J.R. King, A.A. Lacey, and J.L. Vazquez, Persistence of corners in Hele-Shaw flow, Euro. J. Appl. Math., 6(1995), no. 5, 455-490.

[K1] M.K. Korten, Non-negative solutions of $u_{t}=\Delta(u-1)_{+}$: Regularity and uniqueness for the Cauchy problem, Nonl. Anal., Th., Meth., and Appl., 27(1996), 589-603.

[K2] M.K. Korten, A Fatou theorem for the equation $u_{t}=\Delta(u-1)_{+}$, Proc. Am. Math. Soc., 128(1999), no. 2, 439-444. 
[K3] M.K. Korten, On the rectifiability of the free boundary of the one phase Stefan problem, Comm. Anal. and Geom., 13(2005), no. 2, 363-378.

[KM] M.K. Korten and C.N. Moore, The two phase Stefan problem: Regularity of solutions to the Cauchy problem, submitted.

[M] P. Mattila, Geometry of Sets and Measures in Euclidean Spaces, Cambridges studies in advanced mathematics, Vol. 44, Cambridge University Press, (1995).

Department of Mathematics, Kansas State University, Manhattan, KS 66506

E-mail address: blanki@math.ksu.edu, marianne@math.ksu.edu, cnmoore@math.ksu.edu 